\title{
Review of 2 Decades of Aneurysm-Recurrence Literature, Part 2: Managing Recurrence after Endovascular Coiling
}

E. Crobeddu, G. Lanzino, D.F. Kallmes, and H.J. Cloft

\begin{abstract}
SUMMARY: Cerebral aneurysms are treated to prevent hemorrhage or rehemorrhage. Angiographic recurrences following endovascular therapy have been a problem since the advent of this treatment technique, even though posttreatment hemorrhage remains rare. Notwithstanding its unclear clinical significance, angiographic recurrence remains not only a prime focus in the literature but also frequently leads to potentially risky retreatments. The literature regarding aneurysm recurrence following endovascular therapy, spanning 2 decades, is immense and immensely confusing. We review the topic of recurrence following endovascular treatment of cerebral aneurysms in an effort to distill it down to fundamental material relevant to clinical practice.
\end{abstract}

ABBREVIATIONS: BRAT $=$ Barrow Ruptured Aneurysm Trial; CARAT $=$ Cerebral Aneurysm Rerupture After Treatment; $\mathrm{CE}=$ contrast-enhanced; $\mathrm{Cl}=$ confidence interval; ISAT = International Subarachnoid Aneurysm Trial; TOF $=3 \mathrm{D}$ time-of-flight

$\mathrm{T}$ wo decades ago, the development of detachable coils marked the beginning of a revolution in the treatment of cerebral aneurysms. Endovascular coiling proceeded to expand to the point that most ruptured aneurysms in the United States are currently treated with this technique. ${ }^{1}$ While quite successful in improving patient care, endovascular coiling has an "Achilles' heel" in the less-than-perfect long-term protection from rupture noted in a substantial minority of patients. ${ }^{2}$ Aneurysm recurrence has been identified as a problem since the earliest application of this technology. ${ }^{3}$

The problem of recurrence has spawned abundant literature spanning 2 decades. A recent meta-analysis of recurrence rates determined that aneurysm recurrence occurred in $20.8 \%$ of cases (95\% CI, 19.8\%-21.9\%) and retreatment was performed in 10.3\% (95\% CI, 9.5\%-11.0\%). ${ }^{4}$ The recurrence rate after endovascular coiling stands in contrast to the remarkable stability of cerebral aneurysms following surgical clipping. David et $\mathrm{al}^{5}$ reported aneurysm recurrence in only $0.5 \%$ of surgically treated aneurysms at a mean follow-up of 4.4 years. Similarly, Tsutsumi et $\mathrm{al}^{6}$ observed an annual incidence of recurrence after clipping of only $0.26 \%$.

Because aneurysm recurrence represents the primary weakness of endovascular coiling relative to surgery, much of the re-

From the Departments of Radiology (G.L., D.F.K., H.J.C.) and Neurosurgery (E.C., G.L., D.F.K., H.J.C.), Mayo Clinic, Rochester, Minnesota.

Please address correspondence to Harry J. Cloft, MD, PhD, Mayo Clinic, 200 SW First St, Rochester, MN 55905; e-mail: cloft.harry@mayo.edu

-- Indicates open access to non-subscribers at www.ajnr.org

http://dx.doi.org/10.3174/ajnr.A2958 search regarding the endovascular treatment of aneurysms has been directed at the topic of aneurysm recurrence and its prevention. In this review, we summarize this literature regarding the management of recurrence in patients with aneurysms treated with the endovascular approach, with particular attention to the risk of rehemorrhage and the need for imaging surveillance and retreatment.

\section{RECURRENCE LEADING TO REHEMORRHAGE}

The clinical acceptance of endovascular coiling over clipping has been driven by improved outcomes with coiling relative to clipping $^{7,8}$ and by a bias of patients toward less invasive treatment than open surgery. What is really most important clinically is not angiographic recurrence per se but whether the recurrence can cause hemorrhage, especially in patients with ruptured aneurysms. The CARAT study showed that both surgical clipping and endovascular treatment are highly effective in preventing recurrent bleeding from ruptured aneurysms. ${ }^{9}$ These investigators showed that among $>1000$ patients treated between 1996 and 2005 , the risk for rerupture was strongly associated with the degree of aneurysmal occlusion (cumulative risk: 1.1\% for complete occlusion, $2.9 \%$ for $91 \%-99 \%$ occlusion, $5.9 \%$ for $70 \%-90 \%$ occlusion, and $17.6 \%$ for $<70 \%$ occlusion). ${ }^{9}$ Thus, CARAT demonstrated that protection against rebleeding was related to the quality of the aneurysm occlusion, giving perhaps the best scientific evidence that our culture of surveillance for and treatment of aneurysm remnants and recurrences has potential clinical benefit (at least in patients with ruptured aneurysms). In the CARAT study, there was a $2.2 \%$ rerupture rate during the first year after 
coil embolization of ruptured aneurysms, followed by $0.2 \%$ and $0 \%$ in the second and third year, respectively. ${ }^{9}$

The observations of the CARAT investigators are strikingly similar to those reported in the ISAT. In the ISAT, the risk of rebleeding from the target ruptured aneurysm was $2.5 \%$ during the first year after treatment for patients allocated to endovascular treatment. ${ }^{10}$ The annual rebleeding rate after the first year was $0.2 \%$ with a mean follow-up of 4 years. ${ }^{11,12}$ There were more rebleeds from the treated aneurysm in the coiling group than in the clipping group. The cumulative risk of late rebleeding in the coiling group was $1 \%$ after 6 years. The risk of late rebleeding of a coiled aneurysm beyond 6 years is negligible. ${ }^{2}$ This small risk of rebleeding is quite unlikely to offset the initial outcome advantage of endovascular therapy of ruptured aneurysms, except perhaps in patients younger than 40 years of age. ${ }^{13}$

Other recent studies confirm the low risk of rehemorrhage after endovascular coil embolization observed in the CARAT and the ISAT. A multicenter study of 283 coiled ruptured aneurysms with a total follow-up of 1778 patient-years (mean, 6.3 years) showed that the long-term incidence of rebleeding in patients with ruptured aneurysms adequately occluded 6 months after coil embolization was very low $(0.4 \%$ cumulative incidence during 8 years). ${ }^{14}$ In a single-operator experience with coil embolization for ruptured aneurysms spanning 16 years, rehemorrhage occurred in 3 (2.5\%) of 119 aneurysms considered "unstable" on the initial follow-up angiogram and in $3(0.9 \%)$ of 333 aneurysms considered "stable." The hemorrhages occurred at times ranging from 2 months to 9 years following initial therapy. No rebleeding was recorded after the first decade, with 138 patients having $>10$ years of follow-up.

When one assesses the rehemorrhage rates listed above, one must consider that the studies analyzed were conducted during the early evolution of endovascular treatment. There is evidence that with increased operator experience and modern devices, both rates of good outcome and rebleeding rates after endovascular coil embolization have improved. BRAT was a single-center randomized study of coil embolization versus surgery for ruptured intracranial aneurysms. While confirming a better functional outcome at 1 year in patients undergoing coil embolization, the BRAT study showed a better rate of protection than the CARAT or the ISAT because no rebleeding was observed after 2 years in the endovascular cohort. ${ }^{15}$

\section{RISK OF HEMORRHAGE FOLLOWING TREATMENT OF UNRUPTURED ANEURYSMS}

Less is known about the risk of hemorrhage following endovascular therapy of unruptured aneurysms than for ruptured aneurysms. Unruptured aneurysms have a low annual risk of hemorrhage during 5 years without treatment, ${ }^{16}$ and the risk that an unruptured aneurysm treated with coils would rupture is so low that very few will be found even if a large cohort of patients could be followed for many years. A meta-analysis by Naggara et al ${ }^{17}$ found that the annual risk of bleeding after endovascular therapy of unruptured aneurysms was $0.2 \%$, but the clinical follow-up was short, limited to the first 6 months for $76.7 \%(n=1071)$ of reported patients. Immediate angiography following endovascular treatment showed complete obliteration or a small neck rem- nant in $86.1 \%$ of patients. Recurrences were observed in $24.4 \%$ of aneurysms followed up for 0.4-3.2 years, and retreatment was performed in $9.1 \%$ of the cases. ${ }^{17}$ This study considered unruptured aneurysms irrespective of their size, and a review of the studies cited revealed only 1 small aneurysm that went on to rupture after endovascular embolization. ${ }^{18}$ This was a single $8-\mathrm{mm}$ P1-2 junction aneurysm, which may well have been a dissecting pseudoaneurysm rather than a true berry aneurysm. ${ }^{18}$

Other authors have reported a few cases of delayed rupture of previously coiled unruptured aneurysms, but all except the one mentioned above were either large or giant or there was no specification of size. ${ }^{19-25}$ If one considers only small unruptured aneurysms (which represent most aneurysms seen in modern cerebrovascular practices), it is reasonable to assume exceedingly low rupture rates after endovascular treatment based on 2 observations: 1) Despite the vast number of unruptured small aneurysms treated with coil embolization, rupture of a previously unruptured small aneurysm treated with coil embolization is a very rare event and is still considered worth a case report ${ }^{26}$; and 2) there is abundant evidence suggesting a very low incidence of rebleeding after adequate coiling of a small ruptured aneurysm. Thus, it is reasonable to assume a much lower incidence of rupture of an unruptured small aneurysm after coil embolization, given the much more benign natural history of unruptured aneurysms.

\section{IMAGING FOLLOW-UP AFTER ENDOVASCULAR ANEURYSM TREATMENT}

Follow-up imaging after coil embolization of aneurysms is routinely performed in an effort to find recurrences before hemorrhage occurs. Although the data reviewed strongly suggest that hemorrhage is very uncommon after endovascular therapy, the goal of imaging follow-up is to identify those few recurrent aneurysms before they rupture.

\section{Timing of Follow-Up Imaging}

Prolonged imaging follow-up within the first 5-10 years after endovascular treatment is not beneficial in those aneurysms adequately occluded on the 6-month angiographic follow-up because most of these aneurysms continue to stay completely obliterated. ${ }^{27}$

In a large multicenter study from the Netherlands, it was suggested that additional imaging follow-up of aneurysms adequately occluded at 6 months is warranted only for aneurysms of $>10 \mathrm{~mm}$ or for those located at the basilar bifurcation. ${ }^{27}$ The need for routine imaging follow-up beyond 6 months is also questioned by a single-center study of 105 aneurysms, which demonstrated that most coiled ruptured aneurysms that were completely or near-completely occluded at 6 months remained stable at 2 -year follow-up. ${ }^{28}$ The very low incidence of aneurysm rebleeding after the first year observed in the CARAT, ISAT, and other studies ${ }^{14}$ also questions the validity and cost-effectiveness of performing routine follow-up imaging of intracranial aneurysms completely obliterated after 6-12 months.

There certainly is a small chance of aneurysm recurrence, which persists for many years. Although in the ISAT, no rebleeding aneurysms were observed after 6 years from the original treatment, some authors have reported recurrence with the need for 
retreatment up to 10 years after initial treatment. ${ }^{29}$ Stable occlusion on the 6-month angiogram is not an absolute criterion for persistent complete obliteration. In the ISAT, 2 of 988 patients had rehemorrhage from an aneurysm that was completely occluded at 6-month follow-up angiography. ${ }^{12}$ In a series of 452 patients, 3 patients with stable neck remnants at 6 -month angiography went on to rupture 5,6 , and 9 years after the initial treatment. ${ }^{30}$ These observations underscore the need for identifying those aneurysm- and patient-related characteristics predisposing to rupture and/or recurrence after successful endovascular treatment. More research in the future should concentrate on this issue so that the imaging follow-up can be focused only on those patients at risk of recurrence.

It can be argued that imaging screening of patients who have had prior aneurysmal SAH is indicated to detect de novo aneurysm formation. This contention is not supported by existing data. While the incidence of new aneurysm formation in patients with prior aneurysmal SAH is higher than that in the general population, ${ }^{2,5,31}$ the overall incidence is too low to make routine imaging screening cost-effective. ${ }^{32}$

\section{Technique for Follow-up Imaging}

Early imaging follow-up of aneurysms treated with endovascular coil embolization often consists of a catheter cerebral angiography 6-12 months after the original treatment in many units worldwide. Catheter DSA has the advantage of excellent spatial resolution, but with time, MR angiography has gained favor because it is noninvasive. The risk of permanent neurologic complications with conventional angiography is quite low at $0.04 \%-$ $0.07 \%$ in patients with cerebral aneurysms. ${ }^{33,34}$ This low risk of neurologic deficit must be balanced with the risk of missing a recurrence on MR angiography. MR angiography is, however, much more appealing than conventional angiography to patients because of its noninvasive nature, and it is becoming increasingly accepted as a reasonable technique for follow-up imaging of coiled aneurysms. MR angiography also is generally less expensive than DSA. ${ }^{35}$

MR angiography to evaluate aneurysms for recurrence after endovascular coil therapy should be performed with a TOF technique at $1.5 \mathrm{~T}$, which is close enough in sensitivity to DSA to be clinically useful. ${ }^{36}$ Incremental improvements in the detection of aneurysm recurrences may be achieved by imaging with contrastenhancement and/or imaging at $3 \mathrm{~T},{ }^{36-39}$ but imaging at $1.5 \mathrm{~T}$ without contrast is probably sufficient in most cases to detect clinically significant recurrence because TOF MRA at 3T has not been shown to be significantly better in the only study that compared the 2 techniques directly. ${ }^{37}$ A recent meta-analysis showed that TOF-MRA has a sensitivity of $90 \%$ (95\% CI, 79\%-95\%) and a specificity of $95 \%$ (95\% CI, 88\%-98\%) for detecting aneurysm recurrences. CE-MRA had a sensitivity of $92 \%$ and a specificity of $96 \% .{ }^{36}$ Another meta-analysis reported a sensitivity of $83.3 \%$ and a specificity of $90.6 \%$ for TOF-MRA and a sensitivity of $86.8 \%$ and a specificity of $91.9 \%$, for CE-MRA. ${ }^{38}$

In our practice, we perform DSA for initial follow-up imaging of ruptured aneurysms 6 months after treatment because these are the patients at highest risk of bleeding from their coiled aneurysm, and as such, we believe that the highest sensitivity and specificity allow the best decisions regarding the need for additional treatment. In these patients, the first DSA is also helpful in determining the timing of additional imaging follow-up. If the ruptured aneurysm is stable on DSA at 6 months, we think that MR angiography is adequate for any future follow-up. In such cases, future imaging follow-up is tailored to the characteristics of the individual patient and aneurysm. For unruptured aneurysms, we believe that MRA is adequate for follow-up, with DSA indicated to further evaluate any changes seen on MRA. In these patients, MRA is usually performed 1 year following the initial treatment, and for stable aneurysms, another follow-up is recommended in 3-5 years. This practice is based on a pragmatic balance between the evidence presented and the emotional need of patients to have some form of imaging follow-up to rule out aneurysm recurrence and/or de novo aneurysm formation.

\section{MANAGEMENT OF RECURRENCE}

While some aneurysms treated with coils will go on to angiographic recurrence, this can generally be managed safely to avoid bleeding or rebleeding. Ruptured cerebral aneurysms in patients randomized in the $\mathrm{ISAT}^{40}$ had a $17.4 \%$ rate of retreatment after primary endovascular coiling. Approximately half of these were retreated within 3 months of the initial treatment, and the remaining half was retreated later. For the patients with late retreatment, $0.6 \%$ were retreated after rebleeding and $8.3 \%$ were retreated for recurrence on imaging studies. There was no change in the modified Rankin Scale score after further treatment; thus, retreatment after initial coiling had no significant effect on the outcome at 5 years. ${ }^{40}$

The low risk of retreatment is substantiated by a multicenter study of 311 patients with coiled intracranial aneurysms who underwent 352 retreatment procedures for recurrence. In this study, the total combined risk of death and/or permanent major disability was $1.28 \%$ per patient and $1.13 \%$ per procedure. ${ }^{41}$ Another multicenter study of 100 aneurysms in 99 patients requiring additional coiling because of an enlarging remnant and subtotal occlusion reported thromboembolic events in only 3 retreatment procedures with no disabling clinical sequelae. ${ }^{42}$ One bias accounting for at least some of the low risk of retreatment is related to patient selection, with physicians opting for retreatment in technically simpler cases and observation without retreatment for those more technically challenging.

In addition to aneurysm recurrence defined as a worsening of angiographic occlusion of an aneurysm, there is also the related issue of a persistent stable remnant following coil therapy. According to a recent systematic review of the literature, the complete initial occlusion rate is $62.3 \%$ ( $95 \% \mathrm{CI}, 61.2 \%-63.4 \%)$, the near-complete initial occlusion rate is $29.5 \%$ (95\% CI, $28.5 \%$ $30.6 \%$ ), and the incomplete occlusion rate is $8.2 \%$ (95\% CI, $7.5 \%-8.8 \%) .{ }^{4}$ The results of this meta-analysis are remarkably similar to the results of the ISAT trial, which showed $66 \%$ complete angiographic occlusion, $26 \%$ subtotal occlusion or neck remnant, and $8 \%$ incomplete occlusion. ${ }^{12}$ Small remnants very likely pose little risk of hemorrhage to patients, especially in previously unruptured aneurysms. Often the decision to leave a small residual neck is based on the risk/benefit ratio and on the assumption that incomplete treatment is better than no treatment. 
Aneurysm recurrence does not always lead to retreatment. Ferns et $\mathrm{al}^{43}$ reported a series of 124 aneurysms that were incompletely occluded at 6-month imaging. Of 124 aneurysms, 88 were retreated $(71 \%)$, and no complications occurred. Four aneurysms rebled, causing death in 2 patients. Another 4 patients experienced progressive mass effect by growth of the coiled aneurysm, leading to death in 1 . The annual event rate was $1.9 \%$, the annual mortality was $0.7 \%$, and the annual rebleeding rate was $1.0 \%$. In the Hydrocoil: Endovascular Aneurysm Occlusion and Packing Study Trial, only a minority of "major" recurrences were retreated. ${ }^{44}$ A recent study of aneurysm remnants and recurrences demonstrated substantial variability among physicians not only in whether to retreat an aneurysm but also in how to treat it. ${ }^{45}$ Thus, it appears that there is a need for refinement of treatment indications for aneurysm remnants and recurrences.

While one might argue that angiographic recurrences of cerebral aneurysms are a minor problem because such recurrences are rarely associated with hemorrhage, recurrence can actually be a problem for the patient, even if it has not yet occurred because concern for aneurysm recurrence can cause significant psychological stress in patients. In a multicenter study, patients with coiled intracranial aneurysms participating in long-term MRA screening reported anxiety and depression significantly more often than individuals in the general population. ${ }^{46}$ Fear of recurrence after $\mathrm{SAH}$ is related to post-traumatic stress disorder and more pessimism about future life. ${ }^{47}$

In light of these previous studies focused on anxiety and stress, it is important not to create excessive anxiety for patients by imaging for recurrence too frequently or for too long. The patient cares about the appearance of the angiogram only as it correlates with a risk of rebleeding. Moreover, the main reason the patient cares about rebleeding is because it causes death or disability. Language used to describe aneurysm remnants and recurrences and their clinical implications to patients must be carefully crafted so as to not cause undue anxiety. Indeed, it is our observation that if patients are reassured that the risk of bleeding/rebleeding from a small residual/recurrent aneurysm is minimal, then the patient's knowledge about a small remnant does not become a major source of anxiety.

It is important to consider the aneurysm in the context of a patient's overall health. Patients who have survived an SAH have an increased all-cause mortality rate relative to the general population and are more likely to die of causes other than SAH. Getting patients to quit smoking and modify other cardiovascular risk factors is perhaps more important to their overall health and wellbeing than long-term imaging surveillance for aneurysm recurrence. ${ }^{2,48}$ Patients with unruptured aneurysms are also generally less likely to die of SAH than of other causes. ${ }^{16}$ Patients harboring small unruptured aneurysms that have already been coiled probably have a miniscule risk of death or disability from an SAH, so in this group, it is particularly important to consider that the patient almost certainly has more important health matters to consider besides aneurysm recurrence. Similarly, in elderly patients with coiled ruptured aneurysms, surveillance is not indicated because the minimal risk of bleeding is exceeded by the risk of retreatment.

\section{CONCLUSIONS}

During 2 decades, some incremental gains have been made in improving the recurrence rate of aneurysms treated with endovascular coils, but aneurysms occasionally recur. Both patients and physicians consider recurrence to be a suboptimal outcome with some chance of future hemorrhage. Large studies with patients followed for a number of years have taught us that the rates of recurrence and rehemorrhage are not so large as to offset the important clinical benefits of coiling. Imaging follow-up within the first year is the most important step to identify recurrences. Beyond 6 years, follow-up imaging is probably unnecessary for any aneurysms except those already shown on prior imaging to have previously recurred. Patients who have an aneurysm $>10$ $\mathrm{mm}$ or located at the basilar tip or who present at a young age may be a subset of patients who could benefit from occasional imaging surveillance beyond 6 years. With time, we may continue to identify subsets of aneurysms that require increased or decreased surveillance imaging. It is important that we continue to better define the indications for imaging surveillance and retreatment with time so that we can optimally balance avoidance of rebleeding with minimizing unnecessary expense and patient anxiety.

Disclosures: Giuseppe Lanzino_UNRELATED: Other: Educational grants from eV3* and Synthes.* David Kallmes—UNRELATED: Grants/Grants Pending: MicroVention, ${ }^{*}$ eV3, ${ }^{*}$ Micrus, ${ }^{*}$ NFocus, ${ }^{*}$ Sequent, ${ }^{*}$ Penumbra, ${ }^{*}$ Patents (planned, pending, or issued): spine-related patents, ${ }^{\star}$ Royalties: spine fusion royalties, Payment for Development of Educational Presentations: eV3,* CareFusion, ${ }^{*}$ Travel/Accommodations/Meeting Expenses Unrelated to Activities Listed: MicroVention.* Harry Cloft-UNRELATED: Grants/Grants Pending: Mindframe, ${ }^{*}$ Cordis. * Money paid to the institution.

\section{REFERENCES}

1. Smith GA, Dagostino P, Maltenfort MG, et al. Geographic variation and regional trends in adoption of endovascular techniques for cerebral aneurysms. J Neurosurg 2011;114:1768-77

2. Molyneux AJ, Kerr RS, Birks J, et al. Risk of recurrent subarachnoid haemorrhage, death, or dependence and standardised mortality ratios after clipping or coiling of an intracranial aneurysm in the International Subarachnoid Aneurysm Trial (ISAT): long-term follow-up. Lancet Neurol 2009;8:427-33

3. Guglielmi G, Vinuela F, Duckwiler G, et al. Endovascular treatment of posterior circulation aneurysms by electrothrombosis using electrically detachable coils. J Neurosurg 1992;77:515-24

4. Ferns SP, Sprengers ME, van Rooij WJ, et al. Coiling of intracranial aneurysms: a systematic review on initial occlusion and reopening and retreatment rates. Stroke 2009;40:e523-29

5. David CA, Vishteh AG, Spetzler RF, et al. Late angiographic follow-up review of surgically treated aneurysms. J Neurosurg 1999;91:396-401

6. Tsutsumi K, Ueki K, Morita A, et al. Risk of aneurysm recurrence in patients with clipped cerebral aneurysms: results of long-term follow-up angiography. Stroke 2001;32:1191-94

7. Brinjikji W, Rabinstein AA, Nasr DM, et al. Better outcomes with treatment by coiling relative to clipping of unruptured intracranial aneurysms in the United States, 2001-2008. AJNR Am J Neuroradiol 2011;32:1071-75

8. Qureshi AI, Vazquez G, Tariq N, et al. Impact of International Subarachnoid Aneurysm Trial results on treatment of ruptured intracranial aneurysms in the United States: clinical article. J Neurosurg 2011;114:834-41

9. Johnston SC, Dowd CF, Higashida RT, et al. Predictors of rehemorrhage after treatment of ruptured intracranial aneurysms: the Cerebral Aneurysm Rerupture After Treatment (CARAT) study. Stroke 2008;39:120-25 
10. Molyneux A, Kerr R, Stratton I, et al. International Subarachnoid Aneurysm Trial (ISAT) of neurosurgical clipping versus endovascular coiling in 2143 patients with ruptured intracranial aneurysms: a randomised trial. Lancet 2002;360:1267-74

11. Molyneux A, Kerr R, Stratton I, et al. International Subarachnoid Aneurysm Trial (ISAT) of neurosurgical clipping versus endovascular coiling in 2143 patients with ruptured intracranial aneurysms: a randomized trial. J Stroke Cerebrovasc Dis 2002;11:304-14

12. Molyneux AJ, Kerr RS, Yu LM, et al. International Subarachnoid Aneurysm Trial (ISAT) of neurosurgical clipping versus endovascular coiling in 2143 patients with ruptured intracranial aneurysms: a randomised comparison of effects on survival, dependency, seizures, rebleeding, subgroups, and aneurysm occlusion. Lancet 2005;366:809-17

13. Mitchell P, Kerr R, Mendelow AD, et al. Could late rebleeding overturn the superiority of cranial aneurysm coil embolization over clip ligation seen in the International Subarachnoid Aneurysm Trial? J Neurosurg 2008;108:437-42

14. Schaafsma JD, Sprengers ME, van Rooij WJ, et al. Long-term recurrent subarachnoid hemorrhage after adequate coiling versus clipping of ruptured intracranial aneurysms. Stroke 2009;40:1758-63

15. McDougall CG, Spetzler RF, Zabramski JM, et al. The Barrow Ruptured Aneurysm Trial. J Neurosurg 2011 Nov 4. [Epub ahead of print]

16. Wiebers DO, Whisnant JP, Huston J 3rd, et al. Unruptured intracranial aneurysms: natural history, clinical outcome, and risks of surgical and endovascular treatment. Lancet 2003;362:103-10

17. Naggara ON, White PM, Guilbert F, et al. Endovascular treatment of intracranial unruptured aneurysms: systematic review and metaanalysis of the literature on safety and efficacy. Radiology 2010;256:887-97

18. van Rooij WJ, Sluzewski M, Beute GN. Endovascular treatment of posterior cerebral artery aneurysms. AJNR Am J Neuroradiol 2006;27:300-05

19. Terada T, Tsuura M, Matsumoto H, et al. Endovascular treatment of unruptured cerebral aneurysms. Acta Neurochir Suppl 2005;94:87-91

20. Brilstra EH, Rinkel GJ, van der Graaf Y, et al. Treatment of intracranial aneurysms by embolization with coils: a systematic review. Stroke 1999;30:470-76

21. Murayama Y, Tateshima S, Gonzalez NR, et al. Matrix and bioabsorbable polymeric coils accelerate healing of intracranial aneurysms: long-term experimental study. Stroke 2003;34:2031-37

22. Slob MJ, Sluzewski M, van Rooij WJ, et al. Additional coiling of previously coiled cerebral aneurysms: clinical and angiographic results. AJNR Am J Neuroradiol 2004;25:1373-76

23. Kang HS, Han MH, Kwon BJ, et al. Repeat endovascular treatment in post-embolization recurrent intracranial aneurysms. Neurosurgery 2006;58:60-70, discussion 60-70

24. Raymond J, Guilbert F, Weill A, et al. Long-term angiographic recurrences after selective endovascular treatment of aneurysms with detachable coils. Stroke 2003;34:1398-403

25. Eskridge JM, Song JK. Endovascular embolization of 150 basilar tip aneurysms with Guglielmi detachable coils: results of the Food and Drug Administration multicenter clinical trial. J Neurosurg 1998;89:81-86

26. Hodgson TJ, Carroll T, Jellinek DA. Subarachnoid hemorrhage due to late recurrence of a previously unruptured aneurysm after complete endovascular occlusion. AJNR Am J Neuroradiol 1998;19:1939-41

27. Ferns SP, Sprengers ME, van Rooij WJ, et al. Late reopening of adequately coiled intracranial aneurysms: frequency and risk factors in 400 patients with 440 aneurysms. Stroke 2011;42:1331-37

28. Tailor J, Goetz P, Chandrashekar H, et al. Stability of ruptured intracranial aneurysms treated with detachable coils: is delayed follow-up angiography warranted? Br J Neurosurg 2010;24:405-09

29. Gallas S, Januel AC, Pasco A, et al. Long-term follow-up of 1036 cerebral aneurysms treated by bare coils: a multicentric cohort treated between 1998 and 2003. AJNR Am J Neuroradiol 2009;30:1986-92

30. Plowman RS, Clarke A, Clarke M, et al. Sixteen-year single-surgeon experience with coil embolization for ruptured intracranial aneurysms: recurrence rates and incidence of late rebleeding: clinical article. J Neurosurg 2011;114:863-74. Epub 2010 Jul 30

31. Wermer MJ, Buskens E, van der Schaaf IC, et al. Yield of screening for new aneurysms after treatment for subarachnoid hemorrhage. Neurology 2004;62:369-75

32. Wermer MJ, Koffijberg H, van der Schaaf IC. Effectiveness and costs of screening for aneurysms every 5 years after subarachnoid hemorrhage. Neurology 2008;70:2053-62

33. Cloft HJ, Joseph GJ, Dion JE. Risk of cerebral angiography in patients with subarachnoid hemorrhage, cerebral aneurysm, and arteriovenous malformation: a meta-analysis. Stroke 1999;30:317-20

34. Ringer AJ, Lanzino G, Veznedaroglu E, et al. Does angiographic surveillance pose a risk in the management of coiled intracranial aneurysms? A multicenter study of 2243 patients. Neurosurgery 2008; 63: 845-49, discussion 849

35. Schaafsma JD, Koffijberg H, Buskens E, et al. Cost-effectiveness of magnetic resonance angiography versus intra-arterial digital subtraction angiography to follow-up patients with coiled intracranial aneurysms. Stroke 2010;41:1736-42

36. Weng HH, Jao SY, Yang CY, et al. Meta-analysis on diagnostic accuracy of MR angiography in the follow-up of residual intracranial aneurysms treated with Guglielmi detachable coils. Interv Neuroradiol 2008;14(suppl 2):53-63. Epub 2009 Jan 2

37. Kaufmann TJ, Huston J, 3rd, Cloft HJ, et al. A prospective trial of 3T and $1.5 \mathrm{~T}$ time-of-flight and contrast-enhanced MR angiography in the follow-up of coiled intracranial aneurysms. AJNR Am J Neuroradiol 2010;31:912-18

38. Kwee TC, Kwee RM. MR angiography in the follow-up of intracranial aneurysms treated with Guglielmi detachable coils: systematic review and meta-analysis. Neuroradiology 2007;49:703-13

39. Sprengers ME, Schaafsma JD, van Rooij WJ, et al. Evaluation of the occlusion status of coiled intracranial aneurysms with MR angiography at 3T: is contrast enhancement necessary? AJNR Am J Neuroradiol 2009;30:1665-71

40. Campi A, Ramzi N, Molyneux AJ, et al. Retreatment of ruptured cerebral aneurysms in patients randomized by coiling or clipping in the International Subarachnoid Aneurysm Trial (ISAT). Stroke 2007;38:1538-44

41. Ringer AJ, Rodriguez-Mercado R, Veznedaroglu E, et al. Defining the risk of retreatment for aneurysm recurrence or residual after initial treatment by endovascular coiling: a multicenter study. Neurosurgery 2009;65:311-15, discussion 315

42. Renowden SA, Koumellis P, Benes V, et al. Retreatment of previously embolized cerebral aneurysms: the risk of further coil embolization does not negate the advantage of the initial embolization. AJNR Am J Neuroradiol 2008;29:1401-04

43. Ferns SP, Majoie CB, Sluzewski M, et al. Late adverse events in coiled ruptured aneurysms with incomplete occlusion at 6-month angiographic follow-up. AJNR Am J Neuroradiol 2010;31:464-69

44. White PM, Lewis SC, Gholkar A, et al. Hydrogel-coated coils versus bare platinum coils for the endovascular treatment of intracranial aneurysms (HELPS): a randomised controlled trial. Lancet 2011;377:1655-62

45. Daugherty WP, Rad AE, White JB, et al. Observer agreement regarding the necessity of retreatment of previously coiled recurrent cerebral aneurysms. AJNR Am J Neuroradiol 2011;32:566-69

46. Ferns SP, Nieuwkerk PT, van Rooij WJ, et al. Long-term MRA follow-up after coiling of intracranial aneurysms: impact on mood and anxiety. Neuroradiology 2011;53:343-48

47. Noble AJ, Baisch S, Covey J, et al. Subarachnoid hemorrhage patients' fears of recurrence are related to the presence of posttraumatic stress disorder. Neurosurgery 2011;69:323-32, discussion 332-33

48. Huttunen T, von und $\mathrm{Zu}$ Fraunberg M, Koivisto T, et al. Long-term excess mortality of 244 familial and 1502 sporadic one-year survivors of aneurysmal subarachnoid hemorrhage compared with a matched Eastern Finnish catchment population. Neurosurgery 2011;68:20-27 